Management international

Gestiòn Internacional

International Management

\title{
Iniciativa emprenderora e innovación en Barcelona y Montreal. Une comparación del grado de completitud de las políticas
}

\author{
Jaume Valls, Sandra Castillo et Mercè Bernardo
}

Volume 13, numéro hors-série, 2009

Les villes créatives : une comparaison Barcelone - Montréal Creative cities: Comparing Barcelona and Montréal

Ciudades creativas: Una comparicíon Barcelona Montréal

URI : https://id.erudit.org/iderudit/037506ar

DOI : https://doi.org/10.7202/037506ar

Aller au sommaire du numéro

Éditeur(s)

HEC Montréal et Université Paris Dauphine

ISSN

1206-1697 (imprimé)

1918-9222 (numérique)

Découvrir la revue

Citer cet article

Valls, J., Castillo, S. \& Bernardo, M. (2009). Iniciativa emprenderora e innovación en Barcelona y Montreal. Une comparación del grado de completitud de las políticas. Management international / Gestiòn Internacional / International Management, 13, 83-100. https://doi.org/10.7202/037506ar
Résumé de l'article

Dans le cadre des politiques de soutien à la compétitivité, les politiques de soutien à l'innovation et à l'entrepreneurship ont subi une convergence ces dernières années. Le travail utilise la méthodologie du réseau IPREG pour analyser le degré de couverture des politiques et les principales caractéristiques de ces politiques au niveau local. Les cas de Barcelone et Montréal sont ici étudiés : deux villes avec beaucoup de traits en commun identifiées comme pôles de créativité et d'innovation. Dans les politiques de soutien à l'entreprenariat, les résultats montrent une plus grande couverture de la part de Montréal, tandis qu'en ce qui concerne le soutien à l'innovation c'est justement le contraire. Les différences les plus significatives se situent au niveau de trois domaines : le soutien à des collectifs spécifiques, l'éducation et la réduction des barrières.
Tous droits réservés (C) Management international / International Management / Gestión Internacional, 2009
Ce document est protégé par la loi sur le droit d'auteur. L'utilisation des services d'Érudit (y compris la reproduction) est assujettie à sa politique d'utilisation que vous pouvez consulter en ligne.

https://apropos.erudit.org/fr/usagers/politique-dutilisation/ 


\section{Iniciativa emprenderora e innovación en Barcelona y Montreal. Une comparación del grado de completitud de las políticas}

\author{
JAUME VALLS \\ Universitat de Barcelona
}

\author{
SANDRA CASTILLO \\ Universitat de Barcelona
}

\author{
MERCÈ BERNARDO \\ Universitat de Barcelona
}

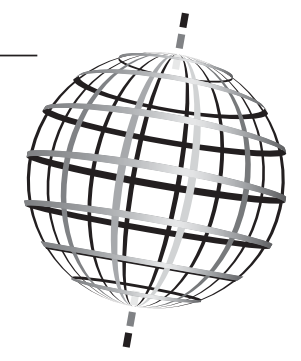

RÉSUMÉ

Dans le cadre des politiques de soutien à la compétitivité, les politiques de soutien à l'innovation et à l'entrepreneurship ont subi une convergence ces dernières années. Le travail utilise la méthodologie du réseau IPREG pour analyser le degré de couverture des politiques et les principales caractéristiques de ces politiques au niveau local. Les cas de Barcelone et Montréal sont ici étudiés : deux villes avec beaucoup de traits en commun identifiées comme pôles de créativité et d'innovation. Dans les politiques de soutien à l'entreprenariat, les résultats montrent une plus grande couverture de la part de Montréal, tandis qu'en ce qui concerne le soutien à l'innovation c'est justement le contraire. Les différences les plus significatives se situent au niveau de trois domaines : le soutien à des collectifs spécifiques, l'éducation et la réduction des barrières.

Mots clés: entreprenariat, innovation, politiques, Barcelone, Montréal, créativité.

\section{Abstract}

Entrepreneurship and innovation policies have developed linkages in competitiveness support policies in last years. This study uses the methodology of IPREG network to analyze the degree of comprehensiveness, and the main characteristics of both policies at the local level. The cases of Barcelona and Montreal are developed, two cities with a great number of similar characteristics, both identified as pools of creativity and innovation. For entrepreneurship support policies, results show a higher degree of comprehensiveness in Montreal while for innovation, the results show the contrary. Main differences among both cities policies are in three areas: support to target groups, education and the elimination of barriers.

Keywords: entrepreneurship, innovation, policies, Barcelona, Montreal, creativity.
RESUMEN

Las políticas de entrepreneuship e innovación han ido convergiendo en los últimos años en el marco de las políticas de apoyo a la competitividad. El presente trabajo utiliza la metodología de la red IPREG para analizar el grado de "completitud", y las principales características de estas dos políticas a nivel local. Se desarrolla el caso de las ciudades de Barcelona y Montreal, dos ciudades con numerosas características similares, identificadas ambas como polos de "creatividad e innovación". En políticas de apoyo a la iniciativa emprendedora los resultados evidencian un mayor grado de completitud en Montreal mientras que en innovación se da el caso contrario. Las diferencias más significativas entre las políticas de las dos ciudades se evidencian en tres áreas: el apoyo a grupos objetivo, la educación y la eliminación de barreras.

Palabras claves: emprendedora, innovación, políticas, Barcelona, Montreal, creatividad.
$\mathrm{E}$ 1 apoyo a la iniciativa emprendedora y a la innovación es hoy en día un tema central de las políticas de competitividad de los países y las regiones. En la últimas dos décadas se ha generalizado el reconocimiento del papel de estas dos áreas en relación con la evolución del crecimiento económico (Drucker, 1985; Lumpkin y Dess, 1996; Lowe y Marriott, 2006; Lee y Kelly, 2008). La políticas de innovación suelen asociarse a tres grandes objetivos (OCDE, 2002a): a) asegurar la generación de nuevo conocimiento e incrementar la efectividad de la inversión pública en innovación; b) mejorar la interacción entre los principales actores del sistema de innovación para potenciar la difusión del conocimiento y de la tecnología y c) establecer los incentivos adecuados para que la innovación en el sector privado transforme conocimiento en valor económico y éxito comercial. Por su parte, las políticas de apoyo a la iniciativa emprendedora tienen por objetivo principal la creación de un entorno y sistema de apoyo que potencie la aparición de nuevos emprendedores, start-ups y las etapas iniciales de crecimiento de nuevas empresas. (Lunsdtrom y Stevenson, 2005)

Estas dos políticas han sido objeto de especial atención desde mediados de los años 90. Por una parte, los gobiernos muestran un creciente interés por las "políticas de entrepreneurship" a medida que se incrementa la evidencia de las relaciones entre las nuevas empresas, la creación de empleo, la productividad, la innovación y el crecimiento económico. El énfasis se sitúa en el "emprendedor individual" y en la creación de un entorno más favorable a la aparición de emprendedores. Como en el caso de la innovación, los enfoques bottom-up adquieren mayor protagonismo y se amplía el alcance de los instrumentos utilizados: programas de apoyo en las escuelas de secundaria, programas orientados a colectivos específicos (mujeres, jóvenes, inmigrantes, etc.), apoyo a redes de Business Angels, etc. Por otra parte, es en esta década cuando las "políticas de ciencia y tecnología" se desplazan hacia las "políticas de innovación" y la visión interactiva del proceso sustituye a la visión lineal. En este contexto, aumenta el número de 
agentes que intervienen en el proceso innovador así como las modalidades de transferencia de tecnología y los instrumentos de las políticas. Finalmente, se incorpora la innovación no-tecnológica a esta visión interactiva del proceso y a las políticas (OCDE, 2002b).

La relevancia adquirida por estas dos políticas va asociada a los avances aportados por la teoría del entrepreneurship y tienen una incidencia significativa en la reconsideración de las políticas de competitividad. El emprendedor transforma conocimiento nuevo en oportunidades comerciales. Realiza una función necesaria para el progreso económico porque apalanca el conocimiento, generado a través de la $\mathrm{I}+\mathrm{D}$ y hace que ese conocimiento termine por convertirse en bienes y servicios comercializados (Acs et al., 2004). Desde este enfoque el emprendimiento se integra con las teorías del desarrollo endógeno. Es en este contexto, en el que numerosos investigadores han descrito un cambio de paradigma en las políticas públicas, en lo que se ha denominado la creación de una "economía emprendedora" (entrepreneurial economy) (Audretsch et $a l, 2006)$. Las políticas públicas orientadas al crecimiento y el empleo que tradicionalmente se habían dirigido a través de los instrumentos macroeconómicos de política fiscal y monetaria, por un lado, y a las ventajas del tamaño y las economías de escala explotadas por las grandes corporaciones, por el otro, han cambiado. Las políticas públicas puestas en marcha en numerosos países (a nivel nacional, pero también regional y local) están enfocándose a la iniciativa emprendedora para reemplazar los empleos perdidos como consecuencia del outsourcing y la globalización, a la vez que se persigue la explotación del potencial existente, derivado de las inversiones en conocimiento que residen en universidades e instituciones educativas y de investigación (Sánchez-Asín, 2008).

El análisis de la evolución de las políticas de apoyo al entrepreneurship y a la innovación, evidencia solapes y un progresivo acercamiento entre las dos áreas. En un contexto de "economía y gestión del conocimiento", crece el interés por el análisis de nuevos temas como por ejemplo: las características del emprendedor, la gestión del capital intelectual o el análisis de la creatividad en las organizaciones. La capacidad emprendedora es un elemento clave de la transferencia de conocimiento en los procesos de comercialización, pero el acceso al conocimiento y la capacidad de transformarlo en una oportunidad no significa necesariamente que pueda llevarse a la práctica ya que un recurso crítico y necesario para llevarlo a cabo es el acceso a la financiación (Audretsch et al., 2006). Todo ello es especialmente visible en las empresas de base tecnológica (Storey y Tether, 1998) y evidencia el papel clave que tienen para su desarrollo las universidades y los parques científicos y tecnológicos.

1. El termino "completitud" es el que han adoptado los autores para traducir el término inglés "comprehensiveness". Una expresión alterna-
El apoyo a la creación de empresas y a la innovación es, hoy en día, un objetivo central de las políticas públicas en los distintos niveles de la administración. La integración de las dos áreas va en aumento y se sitúa en el marco de una nueva generación de políticas de innovación en la que se incrementa la horizontalidad, la coordinación y la integración de la innovación con otras políticas y, simultáneamente, la iniciativa emprendedora y sus indicadores se integran en este marco de políticas (Arundel y Hollanders, 2006). El ejemplo más relevante de esta integración de indicadores lo constituye, bajo nuestro punto de vista, el European Innovation Scoreboard. Está organizado en cinco bloques de indicadores uno de los cuales lleva por título "innovación y entrepreneurship".

El objetivo del presente trabajo es analizar y comparar el entorno de apoyo a la iniciativa emprendedora y a la innovación que existe en Barcelona y Montreal. Se trata, desde nuestro punto de vista, de dos buenos ejemplos de ciudades innovadoras compitiendo a nivel global y con numerosos puntos en común. Para realizar la comparación, se ha llevado a cabo un análisis exploratorio y tentativo siguiendo la metodología de la red IPREG (Innovative Policy Research for Economic Growth) que se basa en la elaboración de los denominados índices de "completitud"1. En el apartado siguiente se sintetiza la metodología utilizada. Posteriormente, se presenta el marco de referencia de ambas ciudades, las principales características de las políticas relacionadas con estás dos áreas de interés y los índices obtenidos. Finalmente, se exponen las principales conclusiones de la comparación realizada.

\section{Políticas de apoyo a la emprendeduría y la innovación}

en la mayoría de países desarrollados, las políticas de apoyo a la creación de empresas se han modificado sustancialmente. El apoyo a la creación de empresas se ha orientado a la figura del emprendedor y se ha pasado gradualmente a aceptar que lo importante es apoyar procesos de creación de empresas guiados por oportunidades y no por necesidades como se había producido en décadas anteriores. Este cambio implica, una conexión muy directa con el apoyo al desarrollo de innovaciones y una creciente importancia del papel de las políticas locales y regionales por la importancia del efecto "proximidad". En los niveles local y regional el entorno de apoyo a las iniciativas emprendedoras y/o innovadoras se configura, frecuentemente, a partir de la convergencia de las políticas de diferentes ámbitos del gobierno, como afirman Acs et. al. (2008), "la creación y el crecimiento de nuevas empresas es claramente un 'positive sum game', no solamente para la localidad, sino para la nación como un todo". En este contexto, las grandes ciudades se han convertido en competidores globales

tiva podría ser "exhaustividad". 


\section{FIGURA 1}

\section{Entorno de Apoyo a la Emprendeduría y la Innovación (a nivel local)}

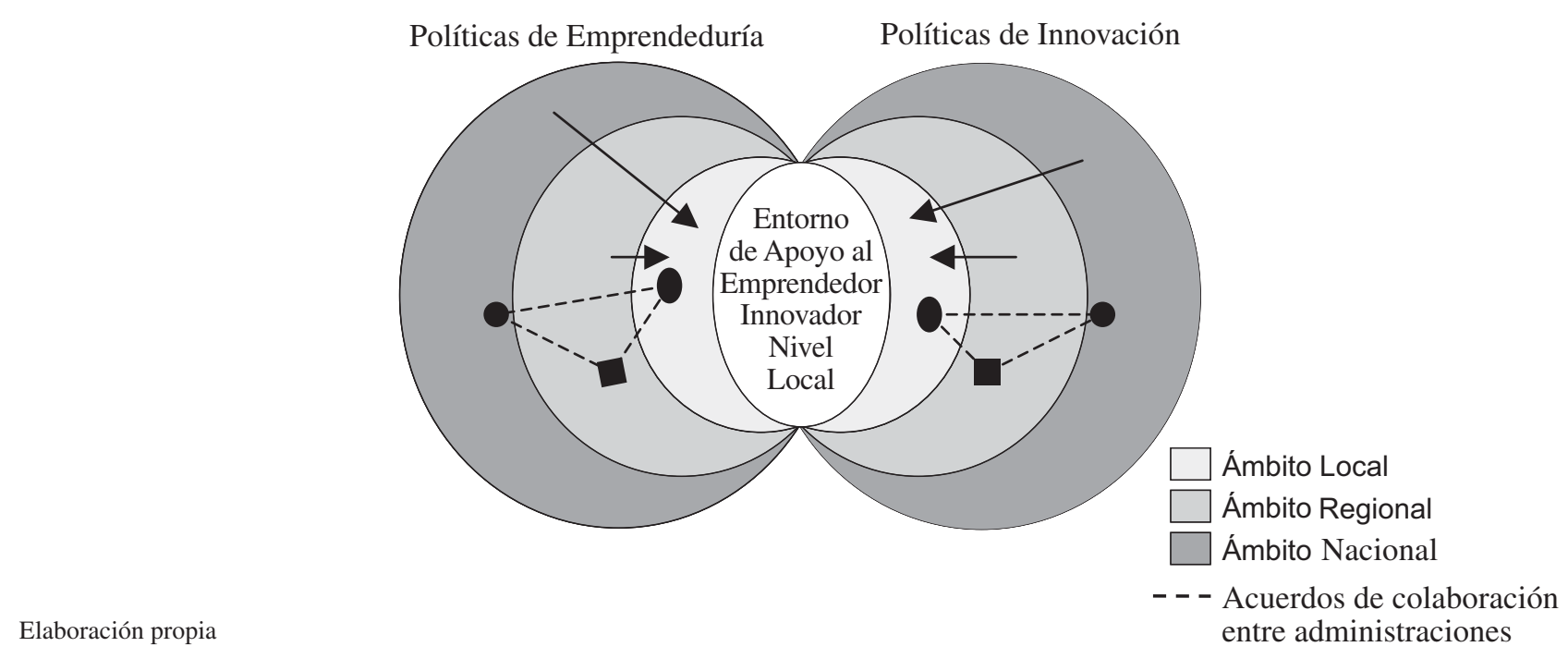

(OCDE, 2006) que desarrollan políticas propias de apoyo a la iniciativa emprendedora y a la innovación en contextos de planificación estratégica muy elaborada en los que aprovechan las interacciones y sinergias de las políticas regionales, nacionales y/o internacionales.

Uno de los puntos clave de la relación entre los diferentes niveles de gobierno es el de las modalidades de colaboración para crear el entorno de apoyo más adecuado a cada localidad: acuerdos, convenios, organización de redes de oficinas de apoyo (figura 1). A modo de ejemplo, un estudio reciente de los mecanismos para vincular las iniciativas de los gobiernos nacionales y regionales, (OECD, 2007) evidencia que en España, el mecanismo más usado es el "convenio de colaboración" entre administraciones. En Canadá, el mecanismo empleado es de tipo contractual y se denomina "acuerdo intergubernamental". La figura 1 representa estas relaciones entre niveles de gobierno y el hecho de que las políticas locales se relacionan y se benefician de las actividades y programas de los otros niveles, dando origen al entorno de apoyo al emprendedor innovador.

\section{El enfoque metodológico de IPREG}

La Red IPREG (Innovative Policy Research for Economic Growth $)^{2}$ fue promovida en 2006 por la Swedish Foundation for Small Business Research (FSF). Se trata de una red internacional, en la que participan investigadores y gestores de políticas públicas de 14 estados europeos. Su objetivo es analizar las políticas de innovación y entrepreneurship, identificando de forma sistemática las medi-

2. En www.ipreg.org está disponible información de las actividades de la red, coordinada por A. Lundström. El informe español del año 2007 das utilizadas por los gobiernos nacionales y los posibles solapamientos, carencias y grados de integración entre las distintas políticas. El marco de análisis de la red parte del trabajo de Lundström y Stevenson (2005) sobre políticas de entreprenurship y lo amplía a la innovación (Lundström et al., 2008). La metodología IPREG se estructura en tres partes: (i) las variables de contexto, (ii) la identificación de los agentes de las políticas y documentos estratégicos y (iii) la elaboración de los indicadores del índice de completitud.

Las variables de contexto se clasifican en tres grupos: a) enfoque general de la política, b) estructura de la política y, c) seguimiento y evaluación. La segunda parte, de identificación, tiene como objetivo principal ilustrar la estructura organizativa del desarrollo e implementación de las políticas, así como sus objetivos. Los índices de completitud, se obtienen teniendo en cuenta siete subáreas de actividad dentro de las políticas: Promoción, Educación, Eliminación de barreras, Financiación, Apoyo, Grupos objetivo e Investigación. La figura 2 esquematiza los niveles de análisis (i) y (iii). La metodología, tiene un carácter marcadamente cualitativo y fundamenta los índices de completitud en un exhaustivo check-list de medidas (ítems) para evaluar la existencia de las mismas dentro de la política y, en algunos casos, su mayor o menor grado de desarrollo.

El análisis de Barcelona y Montreal que se desarrolla en este trabajo tiene un carácter exploratorio y tentativo, utiliza la metodología de la red pero con determinadas simplificaciones que permiten adaptarla para el análisis 


\section{FIGURA 2}

\section{Políticas de emprendeduría e innovación. Áreas de análisis de la metodología IPREG}

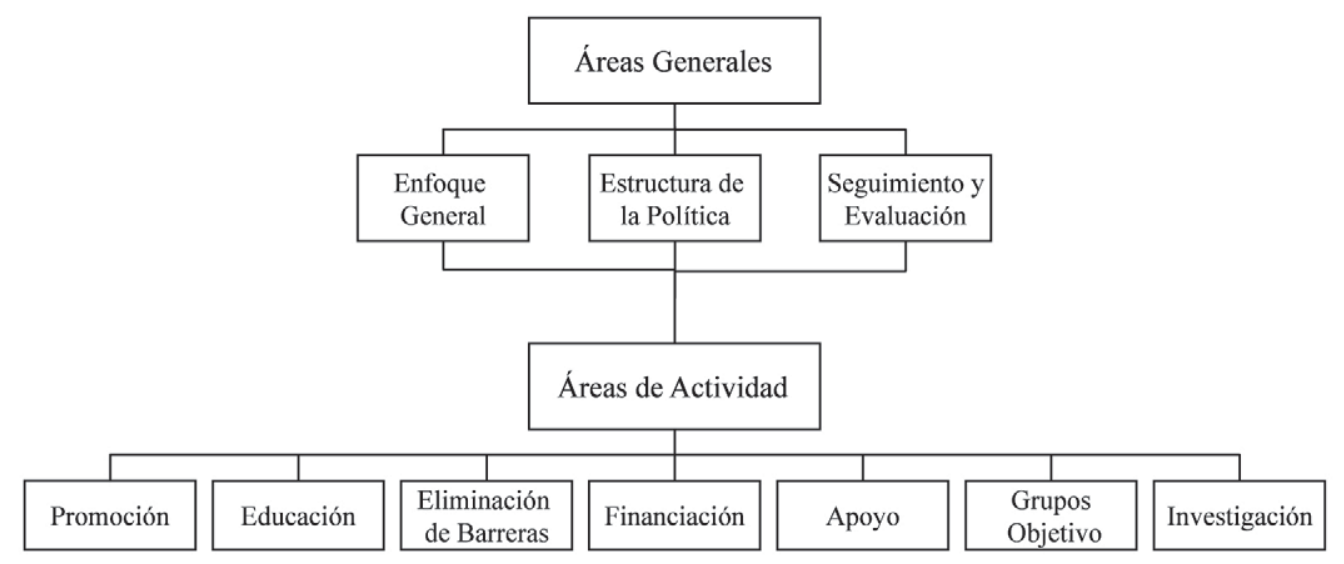

de políticas a nivel de ciudad, ya que la metodología fue diseñada para el análisis de políticas nacionales. La aplicación al nivel local esta supeditada a varias restricciones. Así, en relación con las variables de contexto, es necesario destacar que existe una limitación significativa de fuentes de información. En general, las variables utilizadas son de nivel nacional, y las fuentes de datos utilizadas incluyen Eurostat, Global Entrepreneurship Monitor (GEM), European Innovation Scoreboard (EIS), el Banco Mundial e institutos de estadísticas. El anexo 1 presenta la síntesis de las variables consideradas. Los índices de completitud se construyen a partir de la cantidad de ítems acerca de los cuales se encuentra evidencia en la política analizada (se valora con un 1 si se encuentran evidencias de que existe el ítem y con un 0 en caso contrario). Su medición se realiza en porcentaje, indicando así la cantidad de ítems presentes en la política, con respecto a los que ésta podría involucrar según la metodología. Los índices reflejan la existencia y el grado de completitud de las políticas pero no permiten profundizar en la intensidad con la que se están aplicando determinados instrumentos. A continuación se presentan indicadores generales para contextualizar las dos ciudades a nivel nacional, regional y local.

\section{Indicadores generales}

\section{Indicadores España-Canadá}

Canadá y España están clasificados como países de rentas altas (OECD, 2007). Los dos países poseen estructuras de gobierno en las que se otorga un grado de autonomía significativa a los gobiernos regionales. Canadá es una confederación que cuenta con diez provincias y tres territorios. En virtud de la Ley constitucional de 1867, Québec es aún más descentralizada que otras provincias ya que posee un sistema de leyes mixto, distinto al del resto de provincias. Existen 13 regiones administrativas dentro de esta provincia, una de ellas es la Isla de Montreal, cuya capital lleva su nombre. Por su parte, España se compone actualmente de 17 comunidades autónomas (CCAA) y dos ciudades autónomas. En la Constitución Española de 1978 se fija la cesión del gobierno español de algunas competencias a las CCAA con diferentes grados de descentralización, siendo Cataluña una de las que gestiona un mayor número de competencias. La ciudad de Barcelona es la capital de la región de Cataluña. Dada la existencia de pocos indicadores y estadísticas locales relacionadas con la iniciativa emprendedora y la innovación a nivel local, se ha optado por presentar también algunos indicadores de carácter regional o global (Tabla 1). Es conocido que tanto Quebec como Cataluña poseen un idioma oficial diferente al del conjunto de Canadá o España respectivamente, siendo regiones con una fuerte diferenciación histórica y cultural.

En lo que a iniciativa emprendedora se refiere, según el proyecto Global Entrepreneurship Monitor (GEM , 2005), en términos de crecimiento del PNB en 2005, ambos países tuvieron la misma tasa de crecimiento del 2,8\%. Sin embargo, el National TEA (índice de actividad emprendedora del GEM) fue casi 4 puntos inferior en el caso de España con el 5,4\% de la población involucrada en una actividad emprendedora, mientras que Canadá obtuvo un 9,3\%. En este periodo, Cataluña muestra un TEA ligeramente superior al promedio nacional, mientras que en el caso del Quebec ocurre lo contrario. Otros indicadores relacionados con la iniciativa emprendedora, que permiten comparaciones a nivel internacional son los índices del "Ease of Doing Business Index" (Doing Business, 2008), creado por el Banco Mundial, principalmente para comparar las regulaciones para empresas en los diferentes países. El primero de ellos, National Ease of Starting a Business 
Rank, hace referencia a la facilidad para crear empresas en cada país y tiene en cuenta principalmente, la cantidad de procedimientos, la duración y el costo en los que se incurre al crear una empresa. En relación con esté indicador, únicamente Australia supera a Canadá que ocupa el segundo lugar dentro de 178 países. España en el puesto 118, ocupa uno de los lugares más bajos de Europa, ya que el informe 2008 no encontró reformas en curso para mejorar dichos aspectos. En el caso del indicador relativo a la existencia de las facilidades para las empresas ya creadas, National Ease of Doing Business Rank, aunque la $38^{\mathrm{a}}$ posición de España, sigue siendo inferior en relación a Canadá que se ubica en la séptima, se destacan algunas reformas que está introduciendo en sus regulaciones especialmente orientadas a simplificar procedimientos.

En la tabla 1 se presentan dos indicadores de innovación comparativos a nivel internacional: el ranking de factores nacionales de innovación y el índice agregado (Summary Innovation Index). El primero de ellos pertenece a los indicadores del Global Competitiveness Report (World Economic Forum, 2007). En este caso, se trata del indicador de "sofisticación del negocio e innovación", el cual indica la eficiencia en la producción de bienes y servicios y la innovación tecnológica. Canadá ocupa la posición 17 respecto a 131 países, mientras que España se sitúa en el lugar 31. Por su parte, el Summary Innovation Index (SII) que elabora el European Innovation Scoreboard (Comisión Europea, 2007), tiene una escala de 0 a 1 y proporciona una evaluación comparativa del desempeño en innovación de los estados miembros de la Unión Europea, además de otros países de economías avanzadas. Para saber si un país tiene un índice elevado de desempeño en innovación, se compara su índice con el índice medio para la UE-27. En el informe de 2006 se agruparon los países en cuatro grupos según su índice. Para la UE-27, el índice es del $45 \%$ y existen dos grupos por encima: el de los países considerados líderes en innovación y el de los seguidores en innovación, entre los que se encuentra Canadá con un índice del $44 \%$. Los dos grupos por debajo del índice son los innovadores moderados, en el que se encuentra España con un índice del $31 \%$ y el cuarto grupo son los países que aunque tienen un índice bajo, están mejorando su desempeño.

En adelante, este artículo se centrará en la descripción de los principales elementos del entorno de apoyo a la iniciativa emprendedora y la innovación para los casos concretos de Barcelona y Montreal. Se seguirán las áreas generales y las subáreas de actividad propuestas en el apartado de metodología. Se trata de un análisis tentativo a partir de la información disponible. En muchos casos nos referiremos al ámbito de la "región" de Montreal o de Barcelona, dado el peso de las dos metrópolis para Quebec y Cataluña respectivamente. Este efecto "dimensión” influye, obviamente, en el hecho de que los recursos y las políticas de los distintos ámbitos de gobierno tengan un peso muy significativo en relación con el conjunto de los respectivos territorios.

\section{Indicadores de Barcelona y Montreal}

Barcelona y Montreal han proyectado en las últimas dos décadas una imagen de ciudades innovadoras, de fuerte dinamismo económico y con una apuesta significativa por las actividades industriales y de servicios vinculadas a la alta tecnología. En la planificación estratégica de estas dos ciudades se evidencia que, al igual que sucede en otras

\section{TABLA 1}

\section{Indicadores de entrepreneurship e innovación de España y Canadá}

\begin{tabular}{l|r|r}
\hline & ESPAÑA & CANADÁ \\
\hline $\begin{array}{l}\text { GLOBAL ENTREPRENEURSHIP MONITOR- GEM (2005) } \\
\text { \% Población entre 18 y 64 años, vinculada a una iniciativa emprendedora }\end{array}$ & & \\
- National TEA (Early Stage Entrepreneurial Activity) & $5,4 \%$ & $9,3 \%$ \\
- TEA Regional & Cataluña: 6,1\% & Québec: $9,0 \%$ \\
\hline $\begin{array}{l}\text { DOING BUSINESS (2008) Posición entre 178 países } \\
\text { - National Ease of Starting a Business Rank }\end{array}$ & 118 & \\
- National Ease of doing business Rank & 38 & 7 \\
\hline $\begin{array}{l}\text { GLOBAL COMPETITIVENESS REPORT- GCR (2007-2008) } \\
\text { Posición entre 131 países }\end{array}$ & & \\
- National Innovation Factors Rank & 31 & \\
\hline $\begin{array}{l}\text { EUROPEAN INNOVATION SCOREBOARD -EIS (2007) } \\
\text { - Summary Innovation Index -SII }\end{array}$ & $31 \%$ & \\
\hline \hline
\end{tabular}

Elaboración propia a partir de GEM (2005), índices “Doing Business” (2008) y European Innovation Scoreboard (2007) 


\section{TABLA 2}

Barcelona-Montreal. Demografía e instituciones responsables de las políticas

\begin{tabular}{|c|c|c|}
\hline & BARCELONA & MONTREAL \\
\hline \multicolumn{3}{|l|}{$\begin{array}{l}\text { DEMOGRAFÍA } \\
\text { (habitantes 2006) }\end{array}$} \\
\hline $\begin{array}{l}\text { Población Ciudad } \\
\% \text { Mujeres } \\
\% \text { Hombres } \\
\% \text { Menores de } 35 \text { años } \\
\% \text { Inmigrantes } \\
\text { (Nacidos en otro país) }\end{array}$ & $\begin{array}{r}1.605 .602 \\
52,55 \% \\
47,45 \% \\
39,33 \% \\
17,46 \%\end{array}$ & $\begin{array}{r}1.620 .693 \\
51,83 \% \\
48,17 \% \\
44,30 \% \\
30,25 \%\end{array}$ \\
\hline Población Regional & $\begin{array}{l}\text { Barcelona Provincia: } 5.309 .404 \\
\text { Cataluña: } 7.134 .697\end{array}$ & $\begin{array}{l}\text { Isla de Montreal: } 3.635 .571 \\
\text { Quebec: } 7.546 .131\end{array}$ \\
\hline \multicolumn{3}{|l|}{$\begin{array}{l}\text { INICIATIVA } \\
\text { EMPRENDEDORA }\end{array}$} \\
\hline $\begin{array}{l}\text { Responsable de la Política } \\
\text { en la Ciudad }\end{array}$ & Ayuntamiento de Barcelona & Ayuntamiento de Montreal \\
\hline $\begin{array}{l}\text { Responsable de la Política } \\
\text { Regional }\end{array}$ & $\begin{array}{r}\text { Departamento de Trabajo y, } \\
\text { Departamento de Innovación, } \\
\text { Universidades y Empresa } \\
\text { (Generalitat de Catalunya) }\end{array}$ & $\begin{array}{r}\text { Ministère du Développement } \\
\text { économique, Innovation et Exportation } \\
\text { (Gouvernement du Québec) }\end{array}$ \\
\hline $\begin{array}{l}\text { Responsable de la Política } \\
\text { Nacional }\end{array}$ & $\begin{array}{r}\text { Dirección General de Política de la } \\
\text { Pequeña y Mediana Empresa - } \\
\text { Ministerio de Industria, Turismo y } \\
\text { Comercio (Gobierno de España) }\end{array}$ & $\begin{array}{r}\text { Industry Canada y, } \\
\text { Canada Economic Development for } \\
\text { Québec Regions Department } \\
\text { (Gobierno de Canadá) }\end{array}$ \\
\hline \multicolumn{3}{|l|}{ INNOVACIÓN } \\
\hline $\begin{array}{l}\text { Responsable de la Política } \\
\text { en la Ciudad }\end{array}$ & Ayuntamiento de Barcelona & Ayuntamiento de Montreal \\
\hline $\begin{array}{l}\text { Responsable de la Política } \\
\text { Regional }\end{array}$ & $\begin{array}{r}\text { Departamento de Innovación, Universidad } \\
\text { y Empresa y, } \\
\text { ACC1Ó (Generalitat de Catalunya) }\end{array}$ & $\begin{array}{r}\text { Ministère du Développement } \\
\text { économique, Innovation et Exportation } \\
\text { (Gouvernement du Québec }\end{array}$ \\
\hline $\begin{array}{l}\text { Responsable de la Política } \\
\text { Nacional }\end{array}$ & $\begin{array}{r}\text { Ministerio de Ciencia e Innovación } \\
\text { (Gobierno de España) }\end{array}$ & $\begin{array}{r}\text { Industry Canada y Canada Business } \\
\text { Department } \\
\text { (Gouvernement du Québec) }\end{array}$ \\
\hline
\end{tabular}

Elaboración a partir de Institut d’Estadística de Catalunya, Ajuntament de Barcelona, Cámara de Comercio de Montreal y Statistics Canada.

grandes ciudades y metrópolis del mundo, se utiliza la cooperación publico-privada para potenciar el desarrollo económico mediante el apoyo a la creatividad y la innovación, aspirando a consolidarse como "Regiones Creativas". $\mathrm{Al}$ igual que Montreal en Canadá, Barcelona es la segunda ciudad más poblada de España. Un análisis histórico comparativo del desarrollo urbano de estas dos metrópolis, se puede encontrar en Capel y Linteau (1998).

La tabla 2 presenta los principales aspectos demográficos y gubernamentales de estas dos metrópolis. En los datos demográficos, se puede observar como en Montreal el porcentaje de personas nacidas en otro país casi dobla el porcentaje de Barcelona. Eso se puede explicar por el hecho que el movimiento migratorio en Canadá empezó mucho antes que en España, con orígenes poblacionales muy distintos. Este fenómeno puede condicionar las políticas de apoyo a la iniciativa emprendedora y a la innovación ya que la existencia de dinámicas de inmigración distintas conlleva diferentes niveles de grupos objetivo. 


\section{Apoyo a la iniciativa emprendedora y a la inno- vación en Barcelona}

\section{Políticas de Apoyo a la Iniciativa emprendedora en Barcelona}

En Cataluña, las medidas de apoyo a la creación de empresas $^{3}$, incluyen la promoción, fomento y apoyo del autoempleo (trabajadores autónomos y pequeñas empresas) y el impulso de las empresas de base tecnológica. Estas medidas están enmarcadas dentro de la políticas de Creación de Empleo (Generalitat de Catalunya, 2006) y de la política Industrial (COPCA, 2005; Generalitat de Catalunya, 2006a).

El organismo encargado de la política de creación de empresas es el Departamento de Trabajo a través del Servicio de Creación de Empresas. Este servicio de apoyo, se desarrolla a través de una red de entidades que dan información y asesoran a los emprendedores en la creación de su plan de empresa. La red está compuesta por más de 300 entidades en Cataluña, de las cuales, cerca de 80 están ubicadas en la ciudad de Barcelona. El año 2007 cerca del $20 \%$ de consultas atendidas por el Servicio de Creación de Empresas y el 11\% de las empresas creadas con el apoyo de este organismo, fueron de emprendedores ubicados en Barcelona (Generalitat de Catalunya, 2007a).

Por su parte, el apoyo a la creación de empresas de base tecnológica se desarrolla a través de la agencia de apoyo al desarrollo y la competitividad regional $\mathrm{ACC} \mathrm{O}^{4}$ (que depende del Departamento de Innovación, Universidades y Empresa). Esta agencia ha desarrollado la denominada "red de trampolines tecnológicos" que apoya la creación empresas de base tecnológica a partir de unas oficinas especializadas localizadas en la mayoría de las universidades de la región. Cuatro de estos "trampolines" están localizados en Barcelona.

Tanto el Servicio de Creación de Empresas, como ACC1Ó, poseen mecanismos de colaboración con entidades públicas y privadas que dan apoyo a las iniciativas emprendedoras (cámaras de comercio, universidades, asociaciones sectoriales u otras entidades de carácter empresarial, etc.) ACC1Ó tiene además convenios con el gobierno central para los procesos de apoyo a las empresas de base tecnológica, facilitando el acceso de las empresas generadas en los trampolines a fondos estatales de apoyo del Centro para el Desarrollo Tecnológico Industrial (CDTI).

Finalmente cabe mencionar el papel de Barcelona Activa. Creada en 1986, se trata de la agencia de desarrollo local del Ayuntamiento de Barcelona, dedicada a la promoción del empleo y a la creación y de nuevas empresas (independientemente de su grado de innovación). En estos

3. Una aproximación del marco institucional para la creación de empresas en Cataluña se encuentra en Urbano (2006). veinte años, la agencia ha dado asesoramiento a más de 12.000 proyectos emprendedores, de los cuáles se estima que más del $50 \%$ se han consolidado como empresas y generado cerca de 15.000 puestos de trabajo (Ayuntamiento de Barcelona, 2006). Por su modelo de acompañamiento y los diferentes programas pioneros de apoyo a las personas emprendedoras, ha recibido varios premios y es actualmente identificada como un referente internacional. Barcelona Activa organiza anualmente los "Premios Barcelona Capital Emprendedora". En el ámbito provincial, la Diputación de Barcelona desarrolla también algunas acciones para la promoción del entrepreneurship.

Al utilizar la metodología IPREG adaptada que se ha expuesto en el tercer apartado, el análisis de las áreas generales (representadas en la figura 2) de la política de apoyo a la iniciativa emprendedora en Barcelona evidencia una completitud del $100 \%$, salvo para el caso del área de Seguimiento y Evaluación que es levemente inferior, ya que no se encuentra evidencia suficientemente clara de informes periódicos de dinámica empresarial (entrada, salida, supervivencia, etc.), ni de desagregación de estos datos por género, edad, origen étnico y región. Los índices obtenidos para las subáreas de actividad, se presentan en la figura 3. A continuación se realiza una breve descripción de la evidencia disponible con la que se han elaborado estos índices para la ciudad de Barcelona.

Las medidas relacionadas con la educación, la eliminación de barreras para los emprendedores y los grupos objetivo, son los grupos de medidas que se encuentran en un grado más incipiente en Cataluña. Cabe señalar que en Cataluña, desde el año 2007, se ha instaurado un grupo de trabajo para desarrollar diferentes medidas para la simplificación administrativa de los trámites relacionados con la actividad económica y empresarial (Generalitat de Catalunya, 2007b). Además, conjuntamente con el Ministerio de Trabajo y Asuntos Sociales (Gobierno de España), se han impulsado medidas para mejorar la regulación aplicada a trabajadores autónomos. En materia de Educación, desde el año 2006, con la aprobación de la Ley Orgánica de Educación - LOE (BOE, 2006) el espíritu emprendedor fue reconocido como una competencia básica que pueden adquirir los alumnos en la educación primaria y el bachillerato. Algunas universidades ofrecen asignaturas optativas relacionadas con el entrepreneurship. En Barcelona, poseen este tipo de iniciativas todas las universidades pero, en general, están lejos de los estándares de otros países. La adaptación de los planes de estudio al Espacio Europeo de Educación Superior, en curso, otorgará, sin duda, un mayor relieve a esta competencia. En esta subárea no existen evidencias significativas en primaria ni secundaria, ni la existencia de un programa de formación de educadores.

4. Está denominación de la agencia fue aprobada en 2008 a partir de una reorganización de la estructura del gobierno regional. Entre 1987 y 2008 la denominación fue "Centro de Innovación y Desarrollo Empresarial (CIDEM)" 


\section{FIGURA 3}

\section{Grado de completitud de las subáreas de política de entrepreneurship en Barcelona.}

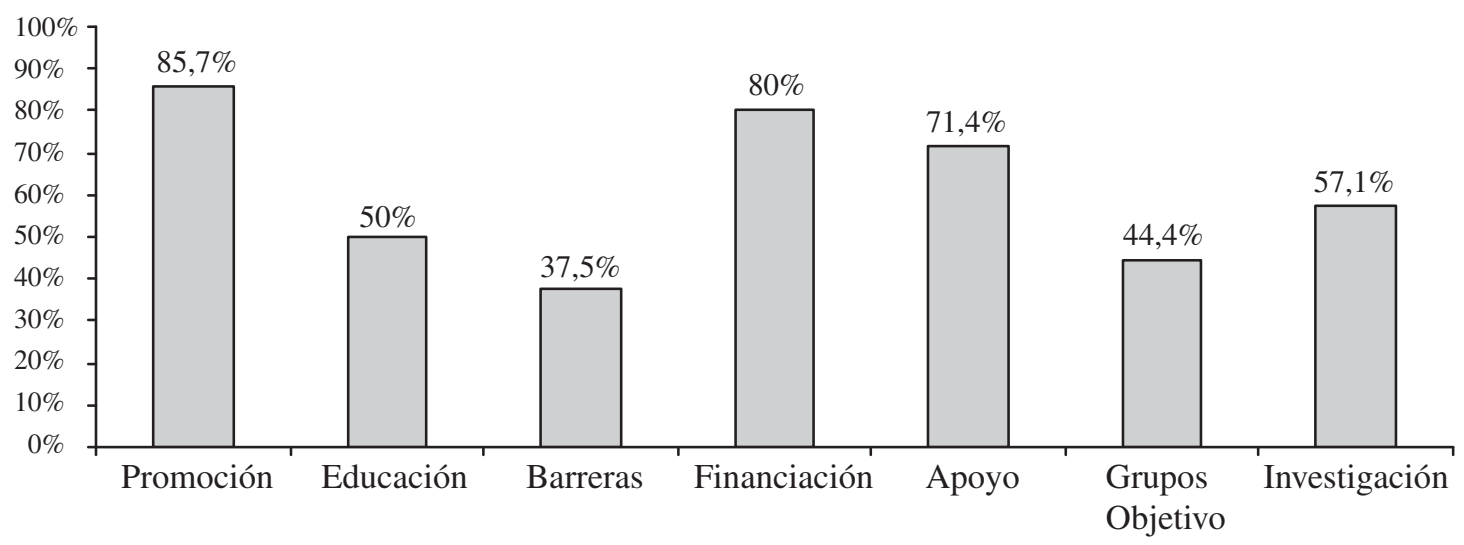

Elaboración Propia.

En el caso de la eliminación de barreras, de los ocho ítems analizados (anexo 1) sólo se ha evidenciado la existencia del punto 1 relativo a facilitar la creación de nuevas iniciativas, la creación de puntos de orientación (punto 6) y las concesiones o reducciones de impuestos (punto 8). Finalmente, para la subárea de políticas orientada a los grupos objetivo, se han evidenciado actuaciones en cuatro de los nueve ítems: mejora de la actividad en ciertos segmentos de la población y programas específicos para mujeres, jóvenes e inmigrantes.

\section{Políticas de innovación en Barcelona}

La política de innovación en Cataluña forma parte de la política más general de investigación e innovación. El ministerio regional responsable de esta política es el Departamento de Empresa, Universidades e Innovación. La política de innovación la lleva a cabo ACC1Ó, agencia a la cual ya nos hemos referido. Las medidas de política de innovación se presentan en un plan de actuación específico, el "Plan de Investigación e Innovación de Cataluña" (PRI), para el período 2005-20085 (GENCAT, 2006a), que trata conjuntamente las políticas de innovación e I+D y que cubre las subáreas propias de la mayoría de planes de este tipo incluyendo la creación de empresas de base tecnológica.

En el Ayuntamiento de Barcelona, además de Barcelona Activa, las áreas de mayor relación con la innovación y la iniciativa emprendedora son las de Educación, y la de Promoción Económica. Vinculado orgánicamente a esta última área está el "Comisionado para la Innovación, la iniciativa empresarial y la investigación”, un cargo político que

5. Documento principal de referencia es el: "Plan de Investigación e Innovación de Catalunya (PRI) 2005-2008" (disponible en http:// www10.gencat.net/pricatalunya). Complementariamente, cabe señalar que en octubre de 2008 se firma el Pacte Nacional per a la Recerca i la coordina iniciativas en relación con la planificación estratégica de la innovación en la ciudad. Existen además comisiones dentro del Consejo Municipal como la de Cultura, Educación y Promoción Social así como la de Promoción Económica, Ocupación y Conocimiento que tiene una regiduría de innovación y ocupación. Dentro de las gerencias sectoriales, está la Gerencia de Promoción Económica que cuenta con un director del Servicio de Innovación. El Programa de Actuación Municipal vigente (2008-2011) (Ayuntamiento de Barcelona, 2007) promociona explícitamente la ciudad como impulsora de la creatividad y la innovación. Un ejemplo de ello, es la reciente difusión del "Mapa de la Innovación" en Barcelona a través de la web"

En cuánto a la financiación para proyectos de innovación, es también ACC1Ó el organismo que pone a disposición de las empresas subvenciones provenientes de los diferentes ámbitos, catalán, español y/o europeo. A nivel español existe el plan InnoEmpresa (2007-2013), cuyo objetivo es poner a disposición de las pequeñas y medianas empresas que lo soliciten, las ayudas necesarias para desarrollar proyectos innovadores que permitan aumentar su competitividad (Ministerio de Industria, Comercio y Turismo, 2007). Hay algunas entidades financieras que proporcionan ayudas para promover la iniciativa emprendedora y la innovación entre los jóvenes.

La promoción de la innovación se hace a través de los Premios de la Generalitat de Catalunya a la competitividad, en los cuales se premian las empresas innovadoras y las que han logrado mayor internacionalización. Además, cada año se celebra el Forum de la Innovación, que reúne más de

Innovació. Un acuerdo promovido por el gobierno regional, firmado por 22 instituciones que marca una estrategia y planificación en innovación e I+D a largo plazo (horizonte 2020).

6. http://mapa.innova.barcelonactiva.cat/ 
tres mil asistentes, en el cual participan figuras reconocidas internacionalmente por su aportación a la mejora de la innovación empresarial. Existe también la red de Innovación creada por la patronal Fomento del Trabajo, para potenciar el intercambio de ideas y buenas prácticas entorno a la innovación ${ }^{7}$. Finalmente, cabe mencionar el programa Innova, una iniciativa pionera de la Universidad Politécnica de Cataluña (UPC) que ayuda a los jóvenes innovadores en la creación de empresas.

Existen numerosas iniciativas de ACC1Ó, de las patronales, de las universidades y de otras organizaciones que inciden en la dinámica innovadora de la ciudad. Entre las existentes en el sector privado cabe señalar Infonomía. Se trata de una organización que detecta y difunde buenas prácticas de innovación, reconocida internacionalmente. Además organiza, desde 2005, iFEST, un encuentro para poner en contacto innovadores de todo el mundo para empezar nuevos proyectos.

El papel de la Asociación Catalana de Parques Científicos (XPcat) ha ido creciendo en importancia en los últimos años. El protagonismo del área de Barcelona en cuanto a parques es muy significativo. Las tres iniciativas catalanas más destacadas están localizadas en el área de Barcelona, a ello hay que añadir la importancia del 22@Barcelona: el distrito de innovación de la ciudad (promovido por el ayuntamiento) que tiene como objetivo la transformación de un espacio industrial en un distrito productivo innovador que ofrece espacios modernos para la concentración estratégica de actividades intensivas en conocimiento.
Siguiendo la metodología IPREG, en el análisis de la Innovación en Barcelona, para las áreas generales los índices de completitud son del 100\% para Enfoque y Estructura, pero de un $80 \%$ para Seguimiento y Evaluación, ya que como en el caso de la iniciativa emprendedora en Barcelona, esto temas tienen aún recorrido de mejora en cuanto a los informes de dinámica empresarial. Los índices obtenidos para las distintas subáreas se presentan en la figura 4, de la cual cabe destacar los índices relativamente débiles en las subáreas de Apoyo, Grupos objetivos e Investigación. En relación al Apoyo, se ha encontrado poca o nula evidencia de la creación de programas de consultoría para nuevas iniciativas, de subsidios para la creación de incubadoras de empresas, de ayudas al desarrollo profesional de asesores ni a la creación de programas de parejas de apoyo. Para los Grupos objetivo no hay evidencias de investigación de las diferentes barreras ni programas específicos para minorías étnicas, desempleados o jubilados y discapacitados. Finalmente, para la Investigación, no se ha evidenciado la existencia de reuniones regulares entre investigadores y gobierno ni un presupuesto específico para programas de investigación relacionada con las políticas de apoyo.

\section{Apoyo a la iniciativa emprendedora e innovación en Montreal}

\section{Apoyo a la iniciativa emprendedaro}

En el modelo canadiense, las medidas relacionadas con la política de apoyo a la pequeña empresa y a la iniciativa emprendedora están íntimamente relacionadas

\section{FIGURA 4}

\section{Grado de completitud de las subáreas de política de Innovación en Barcelona.}

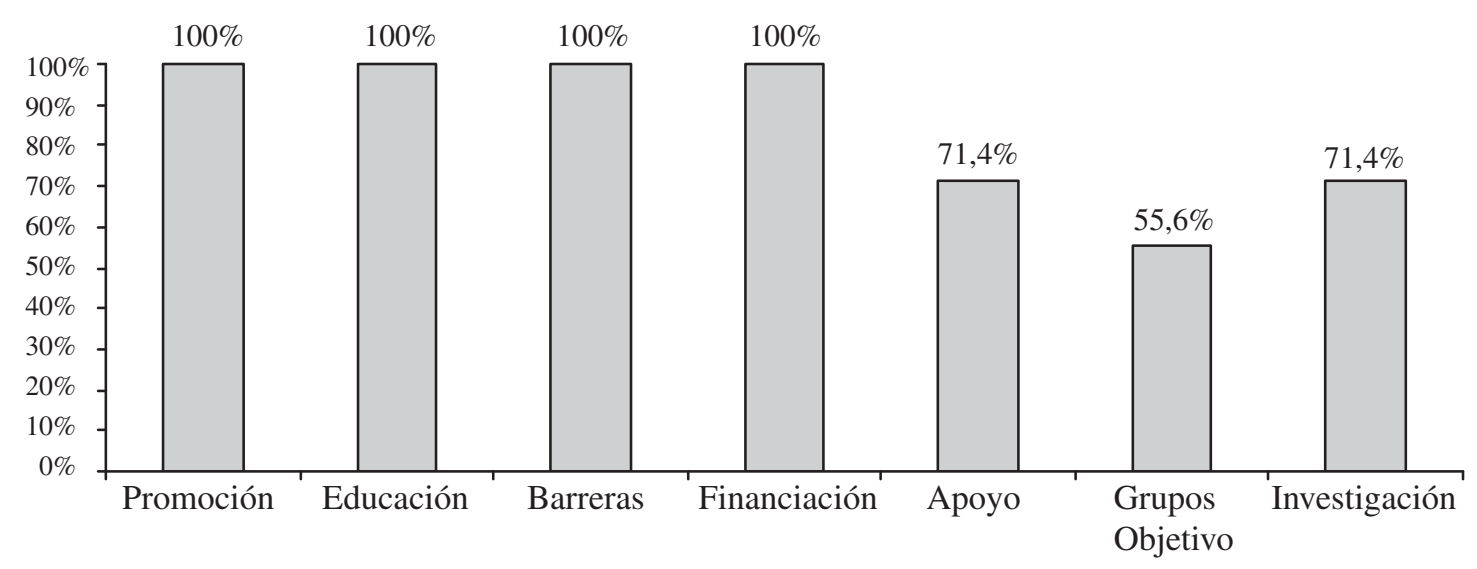

Elaboración Propia. 
con la investigación. El Ministerio de Industria posee una unidad de Recherche et Politique de la Petite Entreprise. En el plan económico "Advantage Canada" (Government of Canada, 2006), se evidencia la importancia de la iniciativa emprendedora para este país, ya que la denominada "Ventaja Emprendedora" constituye uno de los 5 ejes principales de este plan. El presupuesto nacional (Government of Canada, 2007) está diseñado siguiendo las orientaciones de "Advantage Canada" y contiene una serie de medidas presupuestarias específicas para la directriz "Ventaja Emprendedora".

"Entreprise Canada" es un servicio disponible para todo el territorio canadiense, creado con la idea de reducir la complejidad de los trámites relacionados con la empresa. En la provincia de Quebec, este servicio es gestionado y financiado principalmente por el "Développement Économique Canada pour les Régions du Québec", donde el servicio toma el nombre de Infoentrepreneurs y su principal centro se encuentra en Montreal. Para el período 2005-2006, más del $20 \%$ de las solicitudes de información realizadas a "Entreprise Canada" provinieron de emprendedores de la Provincia de Quebec (Government of Canada, 2007a). Por su parte, Infoentrepreneurs-Montréal proporciona acceso a las diferentes instituciones que constituyen el entorno de apoyo a las iniciativas emprendedoras y empresarios.

En la planificación estratégica y presupuestaria de Quebec, se evidencia también el compromiso de los diferentes departamentos de su gobierno con la iniciativa emprendedora, ya que la creación de nuevas empresas y apoyo a las mismas, es uno de las prioridades presentes en dicha planificación ${ }^{8}$. Desde hace 10 años se celebra el concurso de emprendedores en Quebec. Este concurso premia a las mejores iniciativas de cada región de la provincia, principalmente en dos categorías: "Student Entrepreneurship" (mejor idea de negocios de estudiantes desde educación primaria a universitaria) y "Business Creation" (mejores proyectos de negocio en sus etapas iniciales).

En la estrategia de desarrollo económico de la ciudad de Montreal “Reussir@Montréal 2005-2010” (Ville de Montréal, 2005) la creación de empresas es una de las prioridades destacadas. Uno de los principales proyectos de apoyo para emprendedores y empresas consolidadas es el Centro de Innovación para las PyMEs (CIPME), patrocinado por el gobierno de la ciudad y de la provincia de Quebec. Su objetivo es sobre todo la consolidación y crecimiento de empresas de los sectores más tradicionales como los plásticos, metales, muebles y productos petroquími$\cos$, que tienen un rico potencial para el crecimiento y que están siendo amenazados por la creciente globalización. Otro organismo establecido a nivel local, desde 1985, es el Servicio de ayuda a las jóvenes empresas del centro de Montreal (SAJE Montréal Centre). Tiene por misión estimular, favorecer y apoyar a: trabajadores autónomos, la creación y la expansión de PyMEs y la internacionalización de las mismas.

Según Lundström y Stevenson (2002), en general, las políticas de apoyo a la iniciativa emprendedora en Canadá, son de tipo nicho y más concretamente, son políticas de inclusión social. Pretenden disminuir el desempleo o promover la inclusión de ciertos segmentos marginales de la

\section{FIGURA 5}

\section{Grado de completitud de las subáreas de política de entrepreneurship en Montreal.}

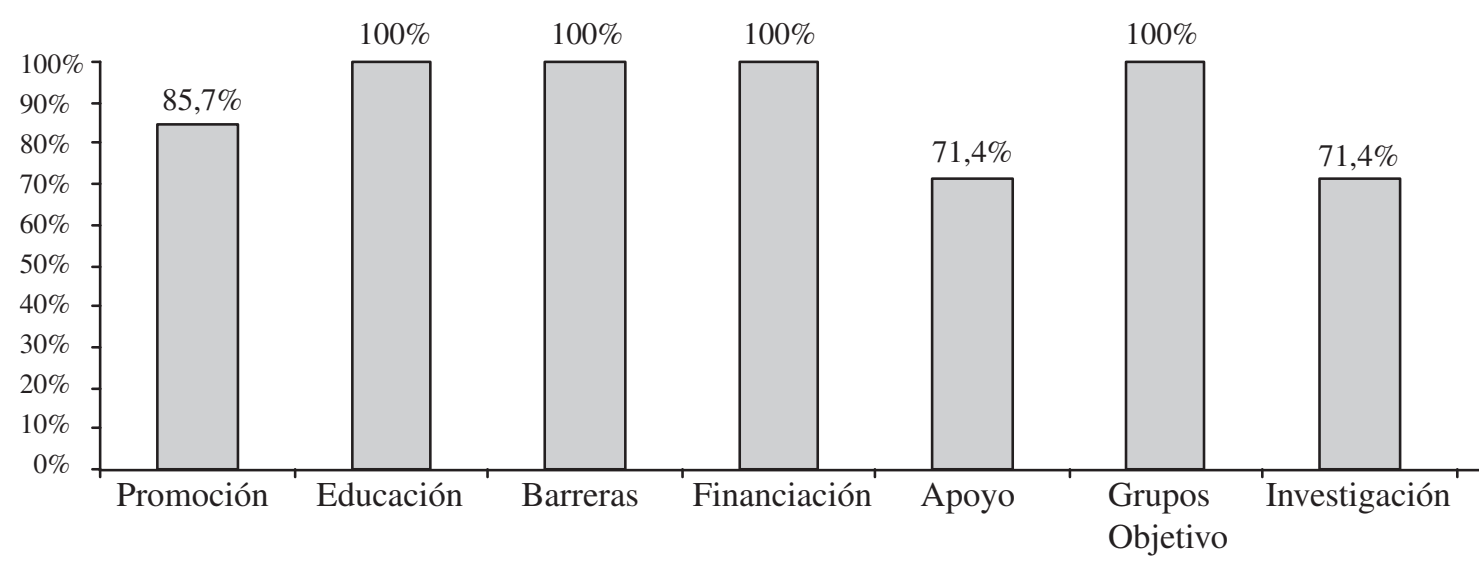

Elaboración Propia.

8. Entre los documentos estratégicos consultados se encuentran: Gouvernement du Québec $(2005,2005 a, 2005 b, 2006)$ y Canada Goverment (2008). 
población. En contraposición con Barcelona, Montreal (en el marco de la Política de la región del Québec) alcanza todos los grupos objetivos posibles: el grupo objetivo que prevalece es el de los jóvenes, seguido por las mujeres y en menor medida por las minorías étnicas, inmigrantes, aborígenes o indios nativos y desempleados.

Los índices obtenidos a través de las evidencias encontradas al estudiar las áreas generales de apoyo a la iniciativa emprendedora que convergen en Montreal, alcanzan el $100 \%$, salvo en el caso del área de Seguimiento y Evaluación, como sucedía en Barcelona, debido a la falta de datos de dinámica empresarial a nivel local. Los índices obtenidos para las subáreas de políticas se resumen en la figura 5. Las subáreas de menor índice de completitud son Promoción, Apoyo e Investigación. Para la actividad de Promoción, no se ha encontrado evidencia de premios a iniciativas según diversidad y grado de madurez. Para el Apoyo, no se ha encontrado evidencia de programas de consultoría o de grupos de apoyo para nuevas iniciativas, ni de subsidios específicos para creación incubadoras o ayudas al desarrollo profesional de asesores. Finalmente, en la subárea de Investigación no se han encontrado evidencias de resultados de investigación en los documentos relacionados con la política o de la realización de reuniones regulares entre investigadores y gobierno.

\section{Políticas de innovación en Montreal}

La política nacional sobre innovación se lleva a cabo desde el Ministerio de Industria, siguiendo el plan Advantage Canada (Government of Canada, 2006). A nivel de Québec, el gobierno regional cuenta con el Ministère du Développement Économique, Innovation et Exportation que ha elaborado el plan "Stratégie Québécoise de la Recherche et de l'Innovation - Un Québec Innovant et
Prospère" (Gouvernement du Québec, 2005c), en el cual se promueve la innovación, se pretende aumentar el número de empresas que invierten en innovación así como mejorar la eficiencia de los mecanismos innovadores. El objetivo es lograr en 2010 que el presupuesto para investigación sea del $3 \%$ del PIB y que aumente la participación financiadora del sector privado. Una de las principales pautas del plan es la de mejorar el apoyo que se le da a la investigación industrial e innovación empresarial.

El sistema nacional de innovación, se puede analizar siguiendo el modelo definido por la OCDE, basado en tres esferas: empresas innovadoras, entorno inmediato y entorno global (Gouvernement du Québec, 2005). En relación con la primera, se observa una ralentización del crecimiento de la I+D, en la segunda, existe una progresión en los acuerdos de cooperación y en la tercera, se observa que la región es una promotora importante de innovación.

A nivel local, el plan Réussir@Montréal 2005-2010 (Ville de Montréal, 2005) diseña la estrategia de desarrollo económico de la ciudad, en el que se enfatiza en el conocimiento, creatividad e innovación (Ville de Montréal, 2007). Se destacan dentro del plan, el conocimiento y la innovación, con acciones como las de promover el desarrollo de capital humano, promover la I+D y el diseño para acelerar la innovación (Montréal Stratégie de l'Innovation) y acelerar el desarrollo del cluster industrial.

La financiación de los proyectos innovadores los lleva a cabo principalmente la Canada Foundation for Innovation (CFI), una corporación creada por el Gobierno de Canadá para financiar la infraestructura de investigación. Su objetivo es fortalecer la capacidad de las universidades, colegios, hospitales e instituciones de investigación sin ánimo de lucro financiándolas. El Gobierno de Québec también aporta

\section{FIGURA 6}

\section{Grado de completitud de las subáreas de política de Innovación en Montreal.}

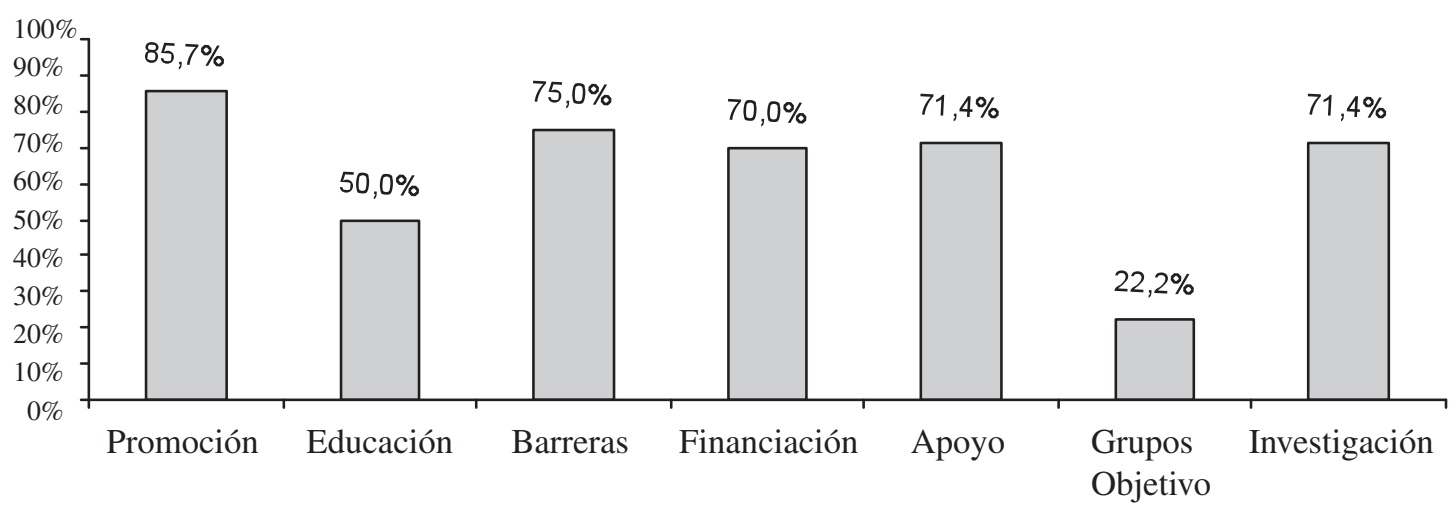

Elaboración Propia. 
ayudas para investigación e innovación (Gouvernement du Québec, 2007). Existen empresas de venture capital. Se puede mencionar, a modo de ejemplo, Innovatech Grand Montreal o BioCapital.

En relación a la promoción y apoyo a la innovación, a nivel nacional existe el Award Plan for Inventors and Innovators Policy dirigido a innovadores que han comercializado su invención con una alta probabilidad de éxito (Federal Partners in Technology Transfer, 2006). A nivel regional existe el premio Lionel-Boulet dirigido a investigadores que han destacado por sus invenciones, innovaciones tecnológicas y científicas, liderazgo científico y han contribuido al crecimiento de la economía de Quebec.
Algunas de las organizaciones que apoyan y promueven la innovación a nivel local son: Montréal Enterprises and Innovation Centre (CEIM): da apoyo a las PYMES para empezar y crecer: Inno-centre ofrece ayuda a las Start-ups y desarrolla alianzas comerciales de tecnologías avanzadas o Technoparc-Montréal, entre otras.

Para las áreas generales de la política, en las tres áreas se han encontrado evidencias que sitúan el índice de completitud en el 100\%. Los índices obtenidos para las subáreas de políticas, se representan en la figura 6. De nuevo, Educación y Grupos objetivo destacan por su bajo índice de completitud respecto al resto de actividades.

\section{FIGURA 7}

\section{Comparación de los índices de las subáreas de política de entrepreneurship (Barcelona - Montreal).}

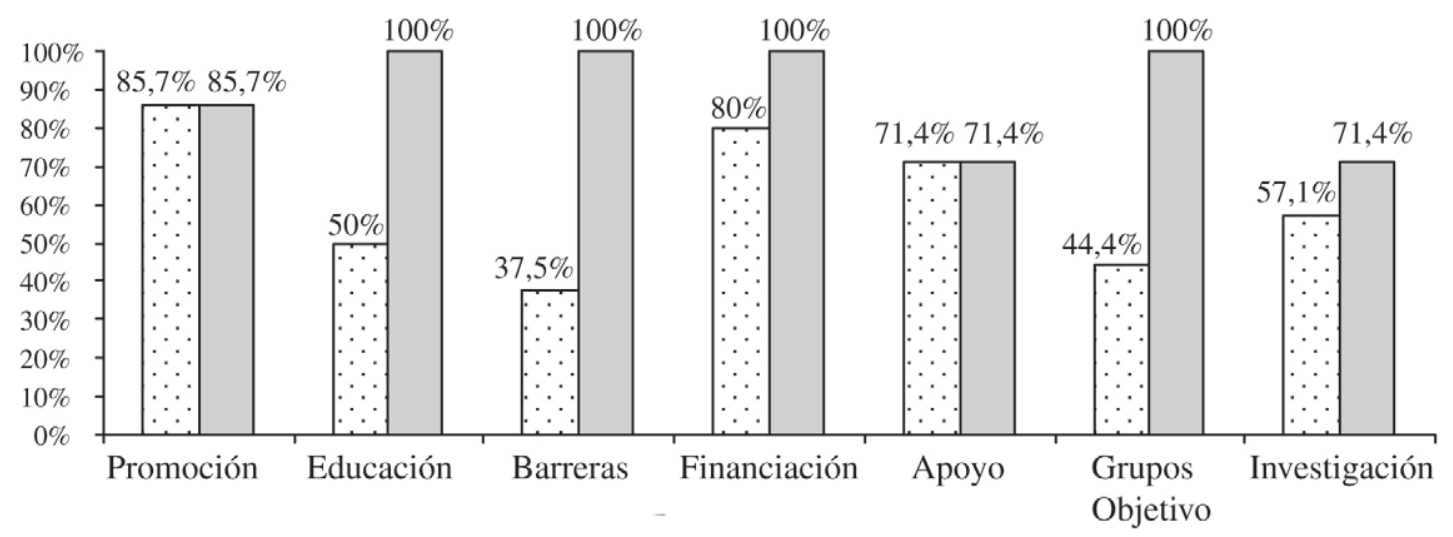

Elaboración Propia

Bacelona $\square$ Montreal

\section{FIGURA 8}

\section{Comparación de los índices de las subáreas de política de innovación (Barcelona - Montreal).}
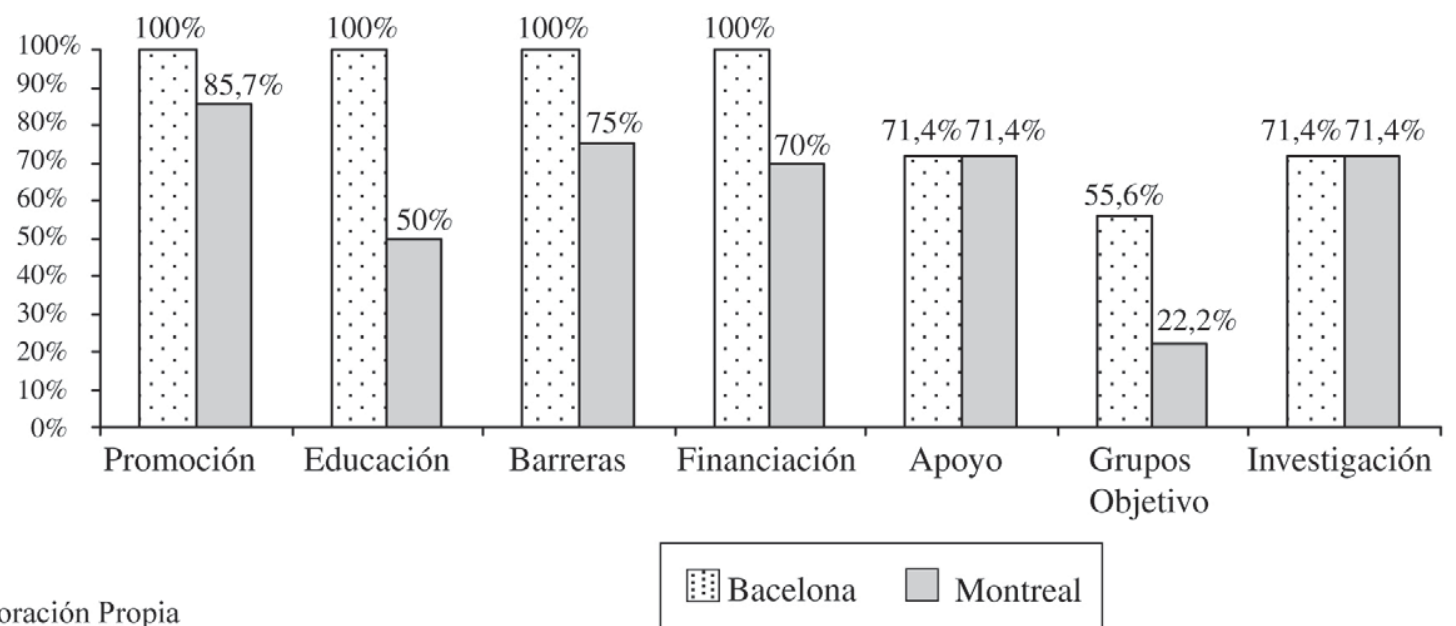
Respecto a Educación, no se han encontrado evidencias de un programa específico de formación de educadores ni de mecanismos para compartir información. Para los Grupos objetivo sólo se han encontrado evidencias para dos de los nueve ítems: la mejora de la actividad innovadora y la existencia de programas específicos para jóvenes.

\section{Comparación de los índices de completitud}

las figuras 7 y 8 presentan la visión comparativa de las subáreas de las políticas. Globalmente el grado de "completitud" de Barcelona es superior en innovación, mientras que para el caso de la iniciativa emprendedora es Montreal quien cuenta con índices más elevados. Cabe remarcar que los resultados son producto de la existencia o no de los ítems y su difusión pública en un determinado contexto temporal. Es decir, puede ser que en algún caso puntual la información sobre el ítem no estuviese disponible en el momento en el que se realizo el trabajo de campo de la investigación. En el apartado siguiente se comentan estos resultados.

\section{Conclusiones}

El análisis de completitud de políticas realizado en este trabajo se basa en una metodología cualitativa de reciente elaboración y difusión que no había sido aplicada a nivel local. En este trabajo hemos realizado esta aplicación que, bajo nuestro punto de vista permite evidenciar algunos aspectos interesantes de la comparación de las políticas de apoyo a la iniciativa emprendedora y a la innovación.

En primer lugar se trataba de evidenciar algunas diferencias significativas entre Barcelona y Montreal al comparar las respectivas políticas de apoyo a la iniciativa emprendedora y a la innovación. Así, en iniciativa emprendedora, las figuras 7 y 8 evidencian un mayor grado de exhaustividad de Montreal en relación con Barcelona, mientras que en innovación se da la situación contraria. En ambos casos, las subáreas en las cuales se evidencian diferencias significativas son tres: Educación, Eliminación de barreras y Grupos objetivo. En el primer caso, la estrecha vinculación del tema con la política educativa regional permitiría matizar los resultados.

Al comparar los índices obtenidos al analizar las políticas de apoyo a la iniciativa emprendedora, Montreal tiene una completitud del 100\% en las áreas de Educación, Eliminación de Barreras y Grupos Objetivo. En el caso de Barcelona parece existir en estos tres grupos un desarrollo de futuras políticas. Es decir, las diferencias principales se producen en tres subáreas en las que se está trabajando en Cataluña, y por extensión en Barcelona: una mayor incorporación de la iniciativa emprendedora en el campo de la docencia, la disminución de barreras para la creación de empresas y la progresiva implementación de un mayor número de instrumentos de apoyo orientados a colectivos muy específicos.
Por otra parte, en relación con el entorno de apoyo a la innovación, las diferencias entre Montreal y Barcelona, se dan principalmente en las subáreas de Educación y Grupos Objetivo. En cuanto a la educación, la evidencia empírica disponible mostraba un nivel inferior de presencia de las actividades del grupo como por ejemplo los mecanismos para compartir información: educadores, seminarios, bases de datos, etc. En cuanto a grupos objetivo, se ha detectado la existencia de una mayor variedad de instrumentos en el caso de Barcelona, aunque en algunos casos, los grupos objetivo de ambas ciudades no coinciden con los que propone la metodología IPREG. También, como se ha observado en los indicadores locales, la composición de la población es diferente entre las ciudades y puede explicar que políticas útiles en Barcelona actualmente pudieran haberlo sido en Montreal anteriormente.

En conjunto, parece que es en el campo de las políticas de entrepreneurship donde ambas ciudades tienen que recorrido en lo que se refiere a alcanzar un mayor grado de completitud. Cabe señalar que, en cuánto al Apoyo, las dos ciudades están al mismo nivel y para educación, en cambio, Barcelona tiene un 100\% de completitud para innovación y un $50 \%$ para iniciativa emprendedrora y en el caso de Montreal la situación es la contraria.

La "completitud" va asociada, en general, a la necesidad de los territorios de dar apoyo a la iniciativa emprendedora y a la innovación con un amplio conjunto de instrumentos y enfoques. Es, en definitiva, un indicador más propio de áreas de elevado desarrollo económico y tecnológico, que además suele ir asociado a elevados grados de integración de las políticas. La integración no ha sido el objetivo de este trabajo pero el análisis de las principales reorganizaciones de los organismos de apoyo y los rediseños de programas recientes de las dos ciudades evidencia que se trata de un tema de plena actualidad.

Los índices de completitud incorporan, en cierto modo, los efectos derivados de las colaboraciones entre los distintos niveles de la administración. Hemos citado algunos ejemplos de instrumentos en los respectivos apartados pero habría muchos más por citar cuya ejecución es posible por las ventajas derivadas de las redes y los sistemas asociados a las aplicaciones de las TIC. Además, por las características de los elevados grados de autonomía política de las dos regiones, las políticas regionales de innovación y ciencia de Quebec y Cataluña cuentan con planes interanuales de apoyo dotados de recursos importantes. El peso económico y demográfico de la metrópolis en relación con la región es muy alto, como lo es la concentración de infraestructuras científico-técnicas y académicas. Por ello la incidencia de estas políticas regionales de apoyo a la innovación es también muy significativa en la ciudad y, en algunos casos, difícil de diferenciar cuando se aborda un análisis como el que aquí se ha planteado.

Un aspecto complementario lo constituyen las políticas de apoyo a sectores relacionados con las nuevas tecnolo- 
gías. Las dos metrópolis han realizado una apuesta por unos pocos sectores: biotecnología, TIC, aeronáutica, nanotecnología, etc. Este tipo de apuestas estratégicas no está bien reflejada en la parrilla de análisis de la metodología IPREG (ver anexo 1) y merecería un tratamiento adicional para completar mejor la visión comparativa aquí planteada. De todos modos este aspecto está parcialmente reflejado en la importancia del apoyo que se da en las dos metrópolis a las empresas de base tecnológica, dado que este tema sí que queda reflejado en la parrilla de análisis.

Las ciudades con indicadores de dinamismo económico destacado, tienen un marco de políticas propio a partir de la acción de los gobiernos locales y una planificación estratégica de la ciudad sofisticada que acaba generando organismos relevantes propios de implementación de la política de la ciudad. Barcelona y Montreal responden a este patrón de comportamiento. Estos organismos, lejos de la visión más convencional del nivel local, adquieren una dimensión más propia de la política regional o nacional. El caso de Barcelona Activa ilustra bien este tema, dado que se fundó como una organización de apoyo a la creación de empresas y está a punto de convertirse en la agencia de "innovación", que integrará el apoyo a la iniciativa emprendedora y a la innovación. Dicho de otro modo, la evolución de las políticas locales de este tipo de ciudades parece reflejar bien la evolución hacia políticas de competitividad integrales en las que progresivamente las políticas de innovación y entrepreneurship se integran juntamente con las políticas de internacionalización.

\section{Bibliografía}

Acs, Z.J., D.B. Audretsch; P. Braunerhjelm, B. Carlsson. (2004) "The missing link: The knowledge filter and entrepreneurship in endogenous growth". En The Knowledge Filter and Entrepreneurship in Endogenous Growth. DP on Entrepreneurship, Growth and Public Policy. \#0805. Max Plank Institute.

Acs, Zoltan, et. al. (2008). "Entrepreneurship and Urban Success: Toward a Policy Consensus". Kansas City: Ewing Marion Kauffman Foundation, 2008.

Audretsch, D.B.; M.C. keilbach; A.E. Lehmann. (2006). "Entrepreneurship and Economic Growth". Oxford University Press, Inc. New York. 2006.

Ayuntamiento de Barcelona (2006). "Barcelona Activa. 20 anys Activant Futur" Ajuntament de Barcelona. Barcelona, 2004.

Ayuntamiento de Barcelona (2007): "Programa d'Actuació Municipal (2008-2011)", Consell Ciutat, Barcelona, España.

Arundel, Anthony y Hugo Hollanders (2006). Trend Chart Methodology Report: "Searching the forest for the trees: "Missing" indicators of innovation" Maastricht
Economic Research Institute on Innovation and Technology (MERIT), July 2006.

BOE (2006). "Ley Orgánica de Educación -LOE”. Gobierno de España. Boletín General del Estado, núm. 106; pág. 17158 - 17207.

CAPel, Horacio y Linteau, Paul-André (1998). "BarcelonaMontréal: Desarrollo urbano comparado". Barcelona: Publicacions Universitat Barcelona. Colección “Geocrítica. Textos de Apoyo", nº 14, 1998.

Comisión Europea (2007). "EUROPEAN INNOVATION SCOREBOARD 2007: Comparative analysis of innovation performance" PRO INNO Europe paper $\mathrm{N}^{\circ} 6$, Luxembourg: Office for Official Publications of the European Communities, 2008, 52 pp.

COPCA (2005). "Pla de Internacionalització de l'Empresa Catalana" 2005-2008. COPCA, Generalitat de Catalunya. Barcelona, 2005.

Doing Business (2008). "Doing Business 2008. Comparing Regulation in 178 Economies". Whasintong D. C.: The World Bank, 2008, 200 pp.

DRUCKER, Peter (1985). "Innovation and Entrepreneurship: Practice and Principles." New York: Harper and Row, 1985, 277 pp.

Federal Partners in Technology Transfer (2006): "10 Years of Leadership in Technology Transfer", Tenth Anniversary Report 1996-2006.

García-Quevedo, Sánchez Asín y Valls-Pasola, J. (2007). Políticas de apoyo a la innovación y a la iniciativa emprendedora. El caso de España. Red IPREG. Madrid: DGPYME, 2007.

GEM (2005). "Global Entrepreneurship Monitor 2005 Excecutive Report". Directores: Maria Minniti, William D. Bygrave and Erkko Autio (Babson College and London Business School), 2005.

GEM (2006). "Global Entrepreneurship Monitor 06 Cataluña - Informe Ejecutivo". Director: Carlos Guallarte (Universitat Autònoma de Barcelona), 2006

Generalitat de Catalunya (2006). "Pla General d'Ocupació a Catalunya" Departament de Treball i Indústria, Barcelona, 2007.

Generalitat de Catalunya (2006a). "Pla de Recerca i Innovació de Catalunya" Generalitat de Catalunya. Barcelona, 2006.

Generalitat de Catalunya (2007). "Pla de Govern 20072010”. Departament de la Presidència, Barcelona, 2007.

Generalitat de Catalunya (2007a). "Informació estadística Dades de l'any 2007" Subdirecció General d'Economia Cooperativa i Creació d'Empreses. Barcelona, 2007.

Generalitat de Catalunya (2007b). "Pla de mesures per a la facilitació de tràmits a l'activitat econòmica i empresarial i la simplificació administrativa”. Barcelona, 2007. 
Government of Canada (2006): "Advantage Canada: Building a Strong Economy for Canadians" Deparment of Fiance, Ottawa, Canada, 2006, 92 pp.

Government of Canada (2007): “The budget Plan 2007 Aspire to a stronger, safer, better Canada”, Department of Finance Canada, Ottawa, Canada, 2007.

Government of Canada (2007a): "Canada Business Annual Report 2005-2006”, Canada Business, Ottawa, Canada, 2007.

Government of Canada (2007b): "Mobilizing Science and Technology to Canada's Advantage", Industry Canada, Ottawa, Canada, 2007.

Government of Canada (2008). Strategic Plan 2008-2011 [en línea] Canada Economic Development for Québec Regions Department [Quebec] www.dec-ced.gc.ca/asp/ general/Ecoute_regions.asp?LANG $=\mathrm{EN}>$ [Consulta: 11 junio 2008]

Gouvernement du Québec (2005). "Plan stratégique 20052008 - Ministère du Développement économique, de l'Innovation et de l'Exportation". Gouvernement du Québec, 2005.

Gouvernement du Québec (2005a). "Plan Stratégique 20052008 - Affaires municipales et Régions " Gouvernement du Québec, 2005.

Gouvernement du Québec (2005b). "Plan Stratégique 20052008 - Ministère de l'Immigration et des Communautés Culturelles" Gouvernement du Québec, 2005.

Gouvernement du Québec (2005c): “Tableau de bord du système d'innovation québécois ". Ministère du Développement économique, de l'Innovation et de l'Exportation . Gouvernement du Québec, 2005.

Gouvernement du Québec (2006). “Un Québec innovant et prospère: Stratégie québécoise de la recherche et de l'innovation" Ministère du Développement économique, de l'Innovation et de l'Exportation . Gouvernement du Québec, 2006.

Gouvernement du Québec (2007): “ Discours sur le budget 2007-2008”, Finances Québec, Gouvernement du Québec, 2007.

INDESCAT. Web de la Estadística oficial de Cataluña : Población [en línea] Generalitat de Catalunya, Ayuntamiento de Barcelona [Barcelona] www.idescat. cat/cat/mapa.html\#poblacio [Consulta: 26 mayo 2008]

LeE, H. y Kelley, D. (2008), "Building dynamic capabilities for innovation: an exploratory study of key management practices", R\&D Management, Vol. 38, Issue 2, pp. 155-168.

Lowe, R. y Marriott, S. (2006), "Enterprise: Entrepreneurship and Innovation. Concepts, Contexts and Commercialization." UK: Butterworth-Heinemann. 2006, 472 pp.
Lumpkin, G. y Dess, G. (1996), "Clarifying the Entrepreneurial Orientation Construct and linking it to Performance", Academy of Management review, 21 (1), pp. 135-172.

Lundström, Anders and Lois Stevenson (2002), "On the road to entrepreneurship policy. Volume 1 of the Entrepreneurship in the future series". Stockholm: Swedish Foundation for Small Business Research, $2002 \ldots$

LundSTRÖM, Anders and Lois Stevenson (2005), Entrepreneurship Policy: Theory and Practice, New York: Springer Science+Business Media, Inc., 2005

LUdSTRÖm A. et al. (2008), IPREG Final Report: "Entrepreneurship and innovation Policies. Analysing measures in European countries" Stockholm: Swedish Foundation for Small Business Research, 2008.

Ministerio de Industria, Comercio y Turismo (2007): “InnoEmpresa (2007-2013)”, Madrid, España, 2007.

Lindholm, Åsa and Lois Stevenson (2005). "Linking Innovation and Entrepreneurship Policy". Stockholm: Swedish Foundation for Small Business Research, 2005 .

OECD (2002). "Comparative Innovation Performance: Countries and Policies for Review" DSTI/STP(2002)43, Paris, OECD, September 2002.

OECD (2002b). "Dynamiser les systèmes nationaux d'innovation" Paris: OCDE, 2002, 100 pp.

OECD (2005). “Governance of Innovation Systems, Vol. 2: Case Studies in Innovation Policy”. Paris: OECD, 2005.

OECD (2006). "Competitive cities in the global economy" Paris: OECD, 2006.

OECD (2006). "Governance of Innovation Systems Volume 3: Case Studies in Cross-Sectoral Policy" Paris: OECD, 2006.

OECD (2007). "Linking Regions and Central Governments: Contracts for Regional Development" Paris: OECD, 2007

SÁnchez-Asín (2008). "Políticas de apoyo a los emprendedores y a la innovación. La economía del entrepreneurship. La financiación". Seminario "Business Angels y iniciativa emprendedora. Consejo Económico y Social de las Islas Baleares. Mallorca, 11 de marzo de 2008.

Statistics Canada, Canada's National Statistical Agency "Comunity Profile" [en línea] Canada Goverment < http://www12.statcan.ca/english/census06/data/profiles/ community/Index.cfm?Lang=E $>$ [Consulta: 26 mayo 2008] 
SolÉParellada, F. Pierre-André, J. (1998): “Imprenditorialità e sviluppo locale". Revista Sviluppo Locale, vol. 7. Pag. 68-93. Torino.

Storey, D. J. y B.S. Tether (1998b): "New technologybased firms in the Euroepan Union: an inroduction". Research Policy (26)9, pp. 933-946.

Urbano, David (2006). "La creación de empresas en Cataluña: organismos de apoyo y actitudes hacia la actividad emprendedora". Col·lecció d'estudis, CIDEM, Generalitat de Catalunya. Barcelona, 2006.

Ville de Montréal (2005). "Stratégie de développement économique 2005-2010 de la Ville de Montréal - reússir@ montréal", Ville de Montréal, Montreal, 2005.

Ville de Montréal (2007). "Economic Report Montréal Agglomeration". Service de la mise en valeur du territoire et du patrimoine, Direction du développement économique et urbain, Ville de Montréal, Montreal, 2007.

World Economic Forum (2007). "Global Competitiveness Report 2007-2008" World Economic Forum, October 2007. 


\section{ANEXO 1}

\section{Políticas locales de apoyo a la innovación y la emprendeduría. Adaptación de la metodología IPREG}

\begin{tabular}{|c|c|c|}
\hline \multicolumn{3}{|l|}{ Ámbito general } \\
\hline Enfoque general de la política & Estructura & Seguimiento y evaluación \\
\hline $\begin{array}{l}\text { 1. Importancia de la política dentro de } \\
\text { la planificación estratégica. } \\
\text { 2. Planes específicos para eliminación } \\
\text { de obstáculos. } \\
\text { 3. Grado de conexión con la política } \\
\text { de PYME. } \\
\text { 4. Grado de conexión con la política } \\
\text { de Innovación. } \\
\text { 5. Objetivos específicos para } \\
\text { incrementar la actividad } \\
\text { emprendedora. } \\
\text { 6. Partida presupuestaria específica. }\end{array}$ & $\begin{array}{l}\text { 1. Organización dentro de la } \\
\text { administración cuya responsabilidad } \\
\text { principal sea la emprendeduría/ } \\
\text { innovación. } \\
\text { 2. Cargo político responsable del } \\
\text { desarrollo del entrepreneurship / } \\
\text { innovación. } \\
\text { 3. Concentración de estas } \\
\text { responsabilidades en un mismo } \\
\text { organismo/responsable. } \\
\text { 4. Estructura centralizada de apoyo a } \\
\text { nuevos emprendedores. }\end{array}$ & $\begin{array}{l}\text { 1. Evaluación y seguimiento de la } \\
\text { cultura/ambiente emprendedor/ } \\
\text { innovador. } \\
\text { 2. Evaluación del impacto de las } \\
\text { medidas propuestas. } \\
\text { 3. Informes de dinámica empresarial } \\
\text { (entrada, salida, supervivencia, etc.) } \\
\text { 4. Desagregación de datos de dinámica } \\
\text { empresarial por género, edad, origen } \\
\text { étnico y región. } \\
\text { 5. Ayudas a (o publicación de) } \\
\text { Investigación relacionada con el } \\
\text { tema. }\end{array}$ \\
\hline
\end{tabular}

\section{Actividades}

1. Promoción $\quad$ 1. Objetivo específico: promocionar la cultura emprendedora/innovadora

2. Eventos para promoción e información

3. Premios a iniciativas destacadas

4. Premios a iniciativas relacionadas con la diversidad (mujeres, jóvenes, minorías) y grado de madurez

\section{Educación}

1.Plan para promover la enseñanza de la emprendeduría/innovación en la educación primaria y secundaria.

2.Programa de entrenamiento a educadores.

3. Eliminación de barreras

1. Objetivo específico: facilitar la creación de nuevas iniciativas.
2. Revisión de los costos relacionados con la creación de nuevas iniciativas.

3. Esfuerzos para examinar las barreras de entrada.

4. Simplificación del proceso de registro de nuevas iniciativas.

\section{Financiación}

1. Objetivo específico: facilitar la financiación de nuevas iniciativas.

2. Revisión de la escasez de financiación para nuevas iniciativas.

3. Esfuerzos para redireccionar la disponibilidad de capital hacia nuevas iniciativas.

4. Programa de microcréditos.

5. Microcréditos específicos para determinados segmentos (mujeres, minorías étnicas y jóvenes).
5. Utilización de medios de comunicación para la promoción.

6. Partida presupuestaria específica para promoción.

7.Esfuerzos para concienciar la población sobre la creación de iniciativas.

3. Mecanismos para compartir información (educadores, seminarios, bases de datos, etc.).

4. Implicación de la administración en el sistema educativo para promover el tema.

5. Número único para el registro y futuros trámites de las nuevas iniciativas.

6. Puntos de orientación donde además de apoyo se puede acceder a toda la información y regulaciones aplicables.

7. Reducción de trámites administrativos para nuevas iniciativas.

8. Reducciones de impuestos.

6. Aval de la administración para facilitar el acceso a créditos.

7. Préstamos de la administración.

8. Programas para acercar inversiones privadas a nuevas iniciativas.

9. Programas para acercar "Business Angels" a nuevas iniciativas.

10.Incentivos a la disponibilidad de "Venture Capital" para nuevas iniciativas 


\begin{tabular}{|c|c|c|}
\hline 5. Apoyo & $\begin{array}{l}\text { 1. Objetivo específico: incrementar el apoyo a } \\
\text { nuevas iniciativas. } \\
\text { 2. Puntos de asesoramiento para facilitar la cober- } \\
\text { tura de las necesidades de nuevas iniciativas. } \\
\text { 3. Suministro de información asistencia y asesoría } \\
\text { a nuevas iniciativas. } \\
\text { 4. Página Web para suministro de información. } \\
\text { 5. Red de centros de asistencia. } \\
\text { 6. Apoyo a la creación de programas de consul- } \\
\text { toría para nuevas iniciativas. } \\
\text { 7. Disponibilidad de ayudas para la formación de } \\
\text { nuevos emprendedores. }\end{array}$ & $\begin{array}{l}\text { 8. Ayudas para la creación de incubadoras de } \\
\text { empresas. } \\
\text { 9. Ayudas para la creación de "Spin-offs". } \\
\text { 10. Ayudas para el desarrollo profesional de as- } \\
\text { esores y agentes de desarrollo económico. } \\
\text { 11.Estándares de gestión para proveedores de } \\
\text { servicios. } \\
\text { 12.Espacios para el intercambio de buenas prác- } \\
\text { ticas entre organismos de apoyo. } \\
\text { 13.Ayudas para la creación de programas de gru- } \\
\text { pos de apoyo y de autoayuda. } \\
\text { 14.Foros y redes donde participen los diferentes } \\
\text { actores (educadores, reguladores, asesores, } \\
\text { políticos, investigadores y emprendedores). }\end{array}$ \\
\hline $\begin{array}{l}\text { 6. Grupos } \\
\text { objetivo }\end{array}$ & $\begin{array}{l}\text { 1. Objetivo específico: mejorar la actividad em- } \\
\text { prendedora/ innovadora en ciertos segmentos } \\
\text { de la población. } \\
\text { 2. Investigación relacionada con la actividad em- } \\
\text { prendedora de los diferentes segmentos de la } \\
\text { población (tasas de creación, supervivencia y } \\
\text { crecimiento). } \\
\text { 3. Investigación de las diferentes barreras y prob- } \\
\text { lemas a los que se enfrentan los diferentes seg- } \\
\text { mentos sociales. }\end{array}$ & $\begin{array}{l}\text { 4. Programas específicos para mujeres. } \\
\text { 5. Programas específicos para jóvenes. } \\
\text { 6. Programas específicos para minorías étnicas. } \\
\text { 7. Programas específicos para desempleados. } \\
\text { 8. Programas específicos para jubilados y dis- } \\
\text { capacitados. } \\
\text { 9. Programas específicos para inmigrantes. }\end{array}$ \\
\hline 7. Investigación & $\begin{array}{l}\text { 1. Apoyo específico a la investigación orientada a } \\
\text { políticas. } \\
\text { 2. Programas especiales de investigación en entre- } \\
\text { preneurship/innovación. } \\
\text { 3. Apoyo a la investigación para la evaluación de } \\
\text { políticas. } \\
\text { 4. Comités de investigadores que asesoren el } \\
\text { planteamiento de medidas referidas a la políti- } \\
\text { ca. }\end{array}$ & $\begin{array}{l}\text { 5. Citación de resultados de investigación con- } \\
\text { cernientes a la política } \\
\text { 6. Reuniones regulares entre investigadores y go- } \\
\text { bierno para discutir resultados recientes de in- } \\
\text { vestigación en entrepreneurship/innovación. } \\
\text { 7. Presupuesto específico para programas de in- } \\
\text { vestigación relacionado con la política }\end{array}$ \\
\hline
\end{tabular}

